\title{
A Novel Technique of Posterolateral Suturing in Thoracoscopic Diaphragmatic Hernia Repair
}

\author{
Yoon Jung Boo ${ }^{1,2}$ Stephan Rohleder ${ }^{2}$ Oliver J. Muensterer ${ }^{2}$ \\ 1 Department of Surgery, Division of Pediatric Surgery, \\ Korea University College of Medicine, Seoul, South Korea \\ 2 Department of Pediatric Surgery, University Medicine of Johannes \\ Gutenberg University, Mainz, Germany \\ Address for correspondence Oliver J. Muensterer, MD, PhD, \\ Department of Pediatric Surgery, University Medicine of Johannes \\ Gutenberg University, Mainz, Germany \\ (e-mail: Oliver.Muensterer@unimedizin-mainz.de).
}

Eur J Pediatr Surg Rep 2017;5:e26-e28.

\begin{abstract}
Background Closure of the posterolateral defect in some cases of congenital diaphragmatic hernia $(\mathrm{CDH})$ can be difficult. Percutaneous transcostal suturing is often helpful to create a complete, watertight closure of the diaphragm. A challenge with the technique is passing the needle out the same tract that it entered so that no skin is caught when the knots are laid down into the subcutaneous tissue. This report describes a novel technique using a Tuohy needle to percutaneously suture the posterolateral defect during thoracoscopic repair of $\mathrm{CDH}$.

Case We report a case of a 6-week-old infant who presented with a $\mathrm{CDH}$ and ipsilateral intrathoracic kidney that was repaired using thoracoscopic approach. The posterolateral part of the defect was repaired by percutaneous transcostal suturing and extracorporeal

Keywords

- congenital diaphragmatic hernia

- thoracoscopy

- Tuohy needle

- suturing technique knot tying. To assure correct placement of the sutures and knots, a Tuohy needle was used to guide the suture around the rib and out through the same subcutaneous tract. The total operative time was 145 minutes and there were no perioperative complications. The patient was followed up for 3 months, during which there was no recurrence.

Conclusion Our percutaneous Tuohy technique for closure of the posterolateral part of $\mathrm{CDH}$ enables a secure, rapid, and tensionless repair.
\end{abstract}

\section{New Insights and the Importance for the Pediatric Surgeon}

Thoracoscopic suturing of the lateral defect of diaphragmatic hernias is challenging. This technique provides a novel, simple alternative of accurately placing secure pericostal sutures subcutaneously through a small needle puncture site.

\section{Background}

Closure of the posterolateral defect in some cases of congenital diaphragmatic hernia (CDH) can be difficult. Percutaneous transcostal suturing is often helpful to create a complete, watertight closure of the diaphragm. ${ }^{1}$ A chal- lenge with the technique is passing the needle out the same tract that it entered so that no skin is caught when the knots are laid down into the subcutaneous tissue. ${ }^{2}$ This report describes a novel technique using a Tuohy needle to percutaneously suture the posterolateral defect during thoracoscopic repair of $\mathrm{CDH}$. received

January 31,2017

accepted after revision

May 22, 2017
DOI https://doi.org/

$10.1055 / \mathrm{s}-0037-1604049$.

ISSN 2194-7619. (c) 2017 Georg Thieme Verlag KG

Stuttgart · New York
License terms

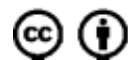




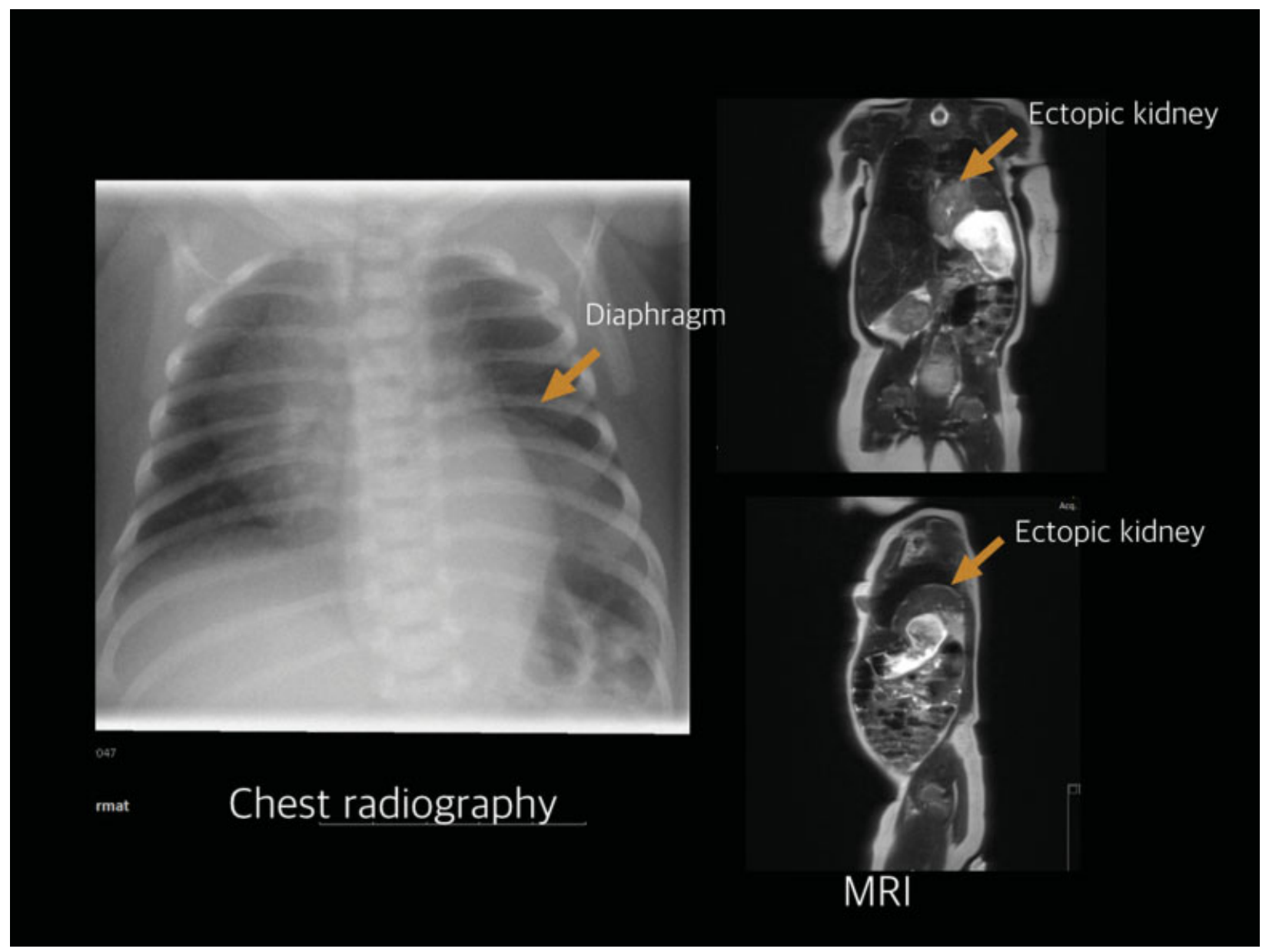

Fig. 1 Chest radiography and magnetic resonance imaging (MRI) examination revealed congenital diaphragmatic hernia (CDH) in the left side and ipsilateral intrathoracic kidney.

\section{Case}

We report a case of a 6 -week-old infant who presented with a $\mathrm{CDH}$ and ipsilateral intrathoracic kidney (-Fig. 1) that was repaired using a thoracoscopic approach. The posterolateral part of the defects was repaired by percutaneous transcostal

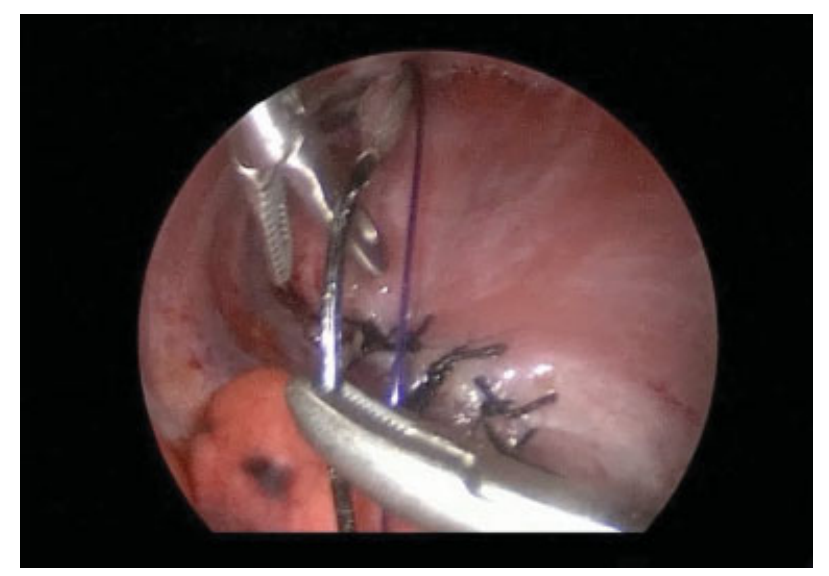

Fig. 2 To assure correct placement of the sutures and knots, a Tuohy needle was used to guide the suture around the rib and out through the same subcutaneous tract. suturing and extracorporeal knot tying. To assure correct placement of the sutures and knots, a Tuohy needle was used to guide the suture around the rib and out through the same subcutaneous tract (-Figs. 2 and 3). The details of this maneuver are presented in - Video 1.

\section{Video 1}

Thoracoscopic percutaneous pericostal suturing to close the lateral defect of congenital diaphragmatic hernia using a Tuohy needle. Online content including video sequences viewable at: www.thieme-connect. com/products/ejournals/html/10.1055/s-00371604049.

\section{Results}

The total operative time was 145 minutes and there were no perioperative complications. The patient was followed up for 3 months, during which there was no recurrence and she did well. 


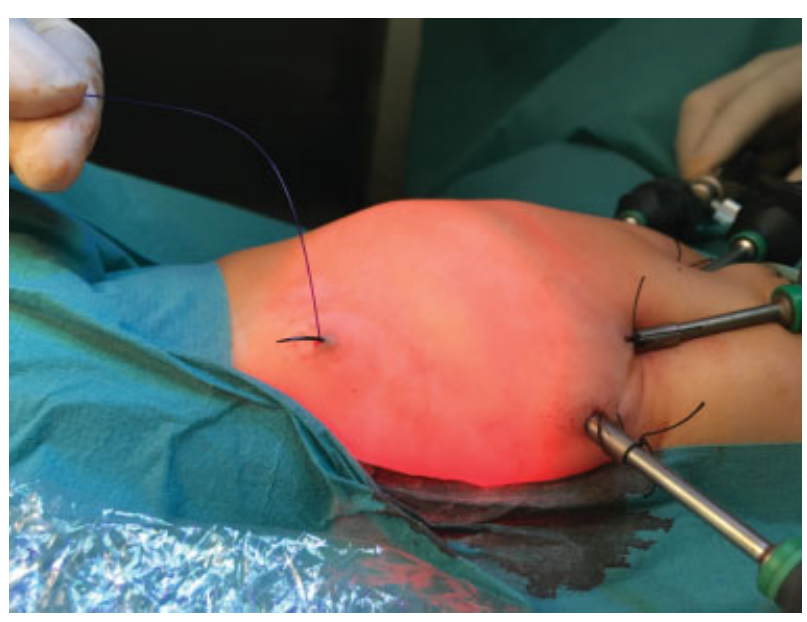

Fig. 3 View of the needle passing out through the same cutaneous incision site guided by Tuohy needle.

\section{Conclusion}

Our percutaneous Tuohy technique for closure of the posterolateral part of $\mathrm{CDH}$ enables a secure, rapid, and tensionless repair.

\section{Conflict of Interests}

None.

\section{References}

1 Li B, Chen BW, Wang QS, Wang BY. Thoracoscopic repair of diaphragmatic hernia in infants: a new modification of the technique. Eur J Pediatr Surg 2016;26(06):519-523

2 Akkoyun I, Keçecioğlu M. A new, easy, and safe suturing technique for laparoscopic repair of Morgagni hernias. J Pediatr Surg 2012; 47(08):1626-1628 